PROCEEDINGS OF THE

AMERICAN MATHEMATICAL SOCIETY

Volume 130, Number 4, Pages 1197-1206

S 0002-9939(01)06147-0

Article electronically published on September 19, 2001

\title{
NOTES ON A $C_{0}$-GROUP GENERATED BY THE LÉVY LAPLACIAN
}

\author{
DONG MYUNG CHUNG, UN CIG JI, AND KIMIAKI SAITÔ
}

(Communicated by Claudia M. Neuhauser)

\begin{abstract}
In this paper we shall give some results on a $C_{0}$-group generated by the Lévy Laplacian and operators approximating that group in the space $\mathcal{L}(\mathbf{E})$ of continuous linear operators defined on a certain locally convex space $\mathbf{E}$ in $(\mathcal{S})^{*}$.
\end{abstract}

\section{INTRODUCTION}

L. Gross 4 and P. Lévy 20] introduced Laplacians on infinite dimensional abstract Wiener space and Hilbert space, respectively. Those Laplacians have been reformulated as differential operators within the framework of white noise distribution theory (see 15, 16, etc.).

Let $(\mathcal{S})^{*}$ be the space of generalized white noise functionals and let $(\mathcal{S})$ be the space of test white noise functionals. The white noise differential operator $\partial_{t}$ is defined to be the Gâteaux differential operator $D_{\delta_{t}}$ in a direction $\delta_{t} \in \mathcal{S}^{*}$, acting on $(\mathcal{S})$. For any $\varphi \in(\mathcal{S})$, the Gross Laplacian $\Delta_{G}$ is given by

$$
\Delta_{G} \varphi=\int_{\mathbf{R}} \partial_{t}^{2} \varphi d t
$$

On the other hand, the Lévy Laplacian $\Delta_{L}$ is given as follows. Let $T$ be a finite interval of $\mathbf{R}$ and let $\left\{\zeta_{k} ; k \in \mathbf{N} \cup\{0\}\right\} \subset \mathcal{S}$ be a complete orthonormal system for $L^{2}(T)$ satisfying the equal density and the uniform boundedness (see [16, 17]). The Lévy Laplacian $\Delta_{L}^{T}$ depending on $T$ is defined by

$$
\Delta_{L}^{T} \Phi=\lim _{n \rightarrow \infty} \frac{1}{n} \sum_{k=0}^{n-1} \widetilde{D}_{\zeta_{k}}^{2} \Phi
$$

if the limit exists in $(\mathcal{S})^{*}$. From now on we fix an interval $T$ and denote $\Delta_{L}^{T}$ by $\Delta_{L}$. This Laplacian acts on some domain in $(\mathcal{S})^{*}$ and disappears on the Hilbert space $\left(L^{2}\right)$ consisting of square-integrable functionals defined on the Gaussian white noise probability space $\left(\mathcal{S}^{*}, \mu\right)$. However $\Delta_{L}$ does not act on the whole space of $(\mathcal{S})^{*}$. So, it is important to decide a domain of the Lévy Laplacian to consider solving a white noise differential equation associated with the Laplacian.

In previous papers [3, 27], we have introduced a domain $\mathbf{E}$ of $\Delta_{L}$ and discussed some relations between a group generated by $\Delta_{G}$ and a group generated by $\Delta_{L}$ in

Received by the editors May 20, 2000 and, in revised form, October 21, 2000.

2000 Mathematics Subject Classification. Primary 60H40.

Key words and phrases. $C_{0}$-group, the Lévy Laplacian, white noise analysis.

(C)2001 American Mathematical Society 
the strong topology of $(\mathcal{S})^{*}$. In $[3$, we also discussed the Cauchy problem associated with $\Delta_{L}$ in the strong topology of $(\mathcal{S})^{*}$ by using the generalized Gross Laplacian $\Delta_{G}(K), K \in \mathcal{L}\left(\mathcal{S}_{\mathbf{C}}^{*}, \mathcal{S}_{\mathbf{C}}\right)$ (see also [2, 27]).

In this article we discuss the above relations in the strong topology of $\mathcal{L}(\mathbf{E})$ by using the generalized Gross Laplacian.

The paper is organized as follows. In Section 2, we summarize some basic definitions and results in the white noise distribution theory. In Section 3, we give a relation between $\Delta_{G}\left(P_{N}\right)$ and $\Delta_{L}$ by the limit theorem in $\mathbf{E}$. In Section 4 , we define groups generated by the Laplacian operators acting on the Hida distributions and give a result that the group generated by $\Delta_{L}$ is approximated by the group $e^{t \Delta_{G}\left(P_{N}\right)}$ in the strong topology of $\mathcal{L}(\mathbf{E})$. In Section 5 , we investigate the fundamental solution of a differential equation associated with the Lévy Laplacian.

\section{Preliminaries}

In this section, we assemble some basic notation of white noise distribution theory following [9, 14, 16, 22]. White noise distribution theory is a Schwartz type distribution theory on the infinite dimensional space $\left(\mathcal{S}^{*}, \mu\right)$, where $\mathcal{S}^{*} \equiv \mathcal{S}^{\prime}(\mathbf{R})$ is the space of tempered distributions and $\mu$ is the standard Gaussian measure such that

$$
\int_{\mathcal{S}^{*}} \exp \{i\langle x, \xi\rangle\} d \mu(x)=\exp \left(-\frac{1}{2}|\xi|_{0}^{2}\right), \xi \in \mathcal{S} \equiv \mathcal{S}(\mathbf{R})
$$

where $\langle\cdot, \cdot\rangle$ is the canonical bilinear form on $\mathcal{S}^{*} \times \mathcal{S}$ and $|\cdot|_{0}$ is the $L^{2}(\mathbf{R})$-norm. Let $A=-(d / d u)^{2}+u^{2}+1$. This is a densely defined self-adjoint operator on $L^{2}(\mathbf{R})$ and there exists an orthonormal basis $\left\{e_{\nu} ; \nu \geq 0\right\} \subset \mathcal{S}$ for $L^{2}(\mathbf{R})$ such that $A e_{\nu}=2(\nu+1) e_{\nu}$. We define the norm $|\cdot|_{p}$ by $|f|_{p}=\left|A^{p} f\right|_{0}$ for $f \in \mathcal{S}$ and $p \in \mathbf{R}$, and let $\mathcal{S}_{p}$ be the completion of $\mathcal{S}$ with respect to the norm $|\cdot|_{p}$. Then the dual space $\mathcal{S}_{p}^{\prime}$ of $\mathcal{S}_{p}$ is the same as $\mathcal{S}_{-p}$ (see 12]). We denote the complexifications of $L^{2}(\mathbf{R}), \mathcal{S}$ and $\mathcal{S}_{p}$ by $L_{\mathbf{C}}^{2}(\mathbf{R}), \mathcal{S}_{\mathbf{C}}$ and $\mathcal{S}_{\mathbf{C}, p}$, respectively.

The space $\left(L^{2}\right)=L^{2}\left(\mathcal{S}^{*}, \mu\right)$ of complex-valued square-integrable functionals defined on $\mathcal{S}^{*}$ admits the well-known Wiener-Itô decomposition

$$
\left(L^{2}\right)=\bigoplus_{n=0}^{\infty} H_{n}
$$

where $H_{n}$ is the space of multiple Wiener integrals of order $n \in \mathbf{N}$ and $H_{0}=\mathbf{C}$. Let $L_{\mathbf{C}}^{2}(\mathbf{R})^{\hat{\otimes} n}$ denote the $n$-fold symmetric tensor product of $L_{\mathbf{C}}^{2}(\mathbf{R})$. If $\varphi \in\left(L^{2}\right)$ is represented by $\varphi=\sum_{n=0}^{\infty} \mathbf{I}_{n}\left(f_{n}\right), f_{n} \in L_{\mathbf{C}}^{2}(\mathbf{R})^{\hat{\otimes} n}$, then the $\left(L^{2}\right)$-norm $\|\varphi\|_{0}$ is given by

$$
\|\varphi\|_{0}=\left(\sum_{n=0}^{\infty} n !\left|f_{n}\right|_{0}^{2}\right)^{1 / 2}
$$

where $|\cdot|_{0}$ also means the norm of $L_{\mathbf{C}}^{2}(\mathbf{R})^{\hat{\otimes} n}$.

For $p \in \mathbf{R}$, let $\|\varphi\|_{p}=\left\|\Gamma(A)^{p} \varphi\right\|_{0}$, where $\Gamma(A)$ is the second quantization operator of $A$. If $p \geq 0$, let $(\mathcal{S})_{p}$ be the domain of $\Gamma(A)^{p}$. If $p<0$, let $(\mathcal{S})_{p}$ be the completion of $\left(L^{2}\right)$ with respect to the norm $\|\cdot\|_{p}$. Then $(\mathcal{S})_{p}, p \in \mathbf{R}$, is a Hilbert space with the norm $\|\cdot\|_{p}$. It is easy to see that for $p>0$, the dual space $(\mathcal{S})_{p}^{*}$ of 
$(\mathcal{S})_{p}$ is given by $(\mathcal{S})_{-p}$. Moreover, for any $p \in \mathbf{R}$, we have the decomposition

$$
(\mathcal{S})_{p}=\bigoplus_{n=0}^{\infty} H_{n}^{(p)}
$$

where $H_{n}^{(p)}$ is the completion of $\left\{\mathbf{I}_{n}(f) ; f \in \mathcal{S}_{\mathbf{C}}^{\hat{\otimes} n}\right\}$ with respect to $\|\cdot\|_{p}$. Here $\mathcal{S}_{\mathbf{C}}^{\hat{\otimes} n}$ is the $n$-fold symmetric tensor product of $\mathcal{S}_{\mathbf{C}}$. We also have $H_{n}^{(p)}=\left\{\mathbf{I}_{n}(f) ; f \in \mathcal{S}_{\mathbf{C}, p}^{\otimes n}\right\}$ for any $p \in \mathbf{R}$, where $\mathcal{S}_{\mathbf{C}, p}^{\hat{\otimes} n}$ is also the $n$-fold symmetric tensor product of $\mathcal{S}_{\mathbf{C}, p}$. The norm $\|\varphi\|_{p}$ of $\varphi=\sum_{n=0}^{\infty} \mathbf{I}_{n}\left(f_{n}\right) \in(\mathcal{S})_{p}$ is given by

$$
\|\varphi\|_{p}=\left(\sum_{n=0}^{\infty} n !\left|f_{n}\right|_{p}^{2}\right)^{1 / 2}, f_{n} \in \mathcal{S}_{\mathbf{C}, p}^{\hat{\otimes} n},
$$

where the norm of $\mathcal{S}_{\mathbf{C}, p}^{\hat{\otimes} n}$ is also denoted by $|\cdot|_{p}$.

The projective limit space $(\mathcal{S})$ of spaces $(\mathcal{S})_{p}, p \in \mathbf{R}$, is a nuclear space. The inductive limit space $(\mathcal{S})^{*}$ of spaces $(\mathcal{S})_{p}, p \in \mathbf{R}$, is nothing but the dual space of $(\mathcal{S})$. The space $(\mathcal{S})^{*}$ is called the space of Hida distributions or generalized white noise functionals. We denote by $\langle\langle\cdot, \cdot\rangle\rangle$ the canonical bilinear form on $(\mathcal{S})^{*} \times(\mathcal{S})$. Then we have

$$
\langle\langle\Phi, \varphi\rangle\rangle=\sum_{n=0}^{\infty} n !\left\langle F_{n}, f_{n}\right\rangle
$$

for any $\Phi=\sum_{n=0}^{\infty} \mathbf{I}_{n}\left(F_{n}\right) \in(\mathcal{S})^{*}$ and $\varphi=\sum_{n=0}^{\infty} \mathbf{I}_{n}\left(f_{n}\right) \in(\mathcal{S})$, where the canonical bilinear form on $\left(\mathcal{S}_{\mathbf{C}}^{\otimes n}\right)^{*} \times\left(\mathcal{S}_{\mathbf{C}}^{\otimes n}\right)$ is also denoted by $\langle\cdot, \cdot\rangle$.

Let $\phi_{\xi}=\exp \{-1 / 2\langle\xi, \xi\rangle\} \exp \langle\cdot, \xi\rangle, \xi \in E_{\mathbf{C}}$. Then $\left\{\phi_{\xi} ; \xi \in E_{\mathbf{C}}\right\}$ spans a dense subspace of $(\mathcal{S})$. The $S$-transform is defined on $(\mathcal{S})^{*}$ by

$$
S[\Phi](\xi)=\left\langle\left\langle\Phi, \phi_{\xi}\right\rangle\right\rangle, \quad \xi \in \mathcal{S}_{\mathbf{C}} .
$$

\section{The generalized Gross Laplacian and the LÉvy Laplacian}

We first introduce the definitions of Laplacian operators following [16] (see also [9, 17]). For any $\varphi=\sum_{n=0}^{\infty} \mathbf{I}_{n}\left(f_{n}\right) \in(\mathcal{S})$, define the Gâteaux derivative $D_{y}$ in any direction $y \in \mathcal{S}^{*}$ by

$$
D_{y} \varphi=\sum_{n=0}^{\infty} n \mathbf{I}_{n-1}\left(\left\langle y, f_{n}\right\rangle\right) .
$$

The white noise differential operator $\partial_{t}$ is defined to be the operator $D_{\delta_{t}}$ acting on $(\mathcal{S})$. For topological linear spaces $E$ and $F$ we denote the set of all continuous linear operators from $E$ into $F$ by $\mathcal{L}(E, F)$. For simplicity we denote $\mathcal{L}(E, E)$ by $\mathcal{L}(E)$. Then, for any fixed $y \in \mathcal{S}^{*}$, the operator $D_{y}$ is in $\mathcal{L}((\mathcal{S}))$. Therefore the differentiation $\partial_{t}$ is in $\mathcal{L}((\mathcal{S}))$ and its adjoint operator $\partial_{t}^{*}$ is in $\mathcal{L}\left((\mathcal{S})^{*}\right)$. For any $\eta \in \mathcal{S}$, the differentiation $D_{\eta}$ has a unique extension to a continuous linear operator $\widetilde{D}_{\eta}$ in $\mathcal{L}\left((\mathcal{S})^{*}\right)$ (for more details, see [16] or [22]).

For any $K \in \mathcal{L}\left(\mathcal{S}_{\mathbf{C}}^{*}, \mathcal{S}_{\mathbf{C}}\right)$, the generalized Gross Laplacian $\Delta_{G}(K)$ is defined by

$$
\Delta_{G}(K) \Phi=\sum_{n=0}^{\infty} \mathbf{I}_{n}\left((n+2)(n+1) \tau(K) \hat{\otimes}_{2} F_{n+2}\right),
$$

for $\Phi=\sum_{n=0}^{\infty} \mathbf{I}_{n}\left(F_{n}\right) \in(\mathcal{S})^{*}$, where $\tau(K)$ is defined by a generalized function in $\left(\mathcal{S}_{\mathbf{C}} \otimes \mathcal{S}_{\mathbf{C}}\right)^{*}$ such that

$$
\langle\tau(K), \xi \otimes \eta\rangle=\langle K \xi, \eta\rangle,
$$


and $\tau(K) \hat{\otimes}_{2} F_{n+2}$ is the right contraction (see [22]). The Laplacian $\Delta_{G}(K)$ is a continuous linear operator in $\mathcal{L}\left((\mathcal{S})^{*}\right)$.

Let $T$ be a finite interval of $\mathbf{R}$ and let $\left\{\zeta_{k} ; k \in \mathbf{N} \cup\{0\}\right\} \subset \mathcal{S}$ be a complete orthonormal system for $L^{2}(T)$ satisfying the equal density and the uniform boundedness (see [16, 17). The Lévy Laplacian $\Delta_{L}^{T}$ depending on $T$ is defined by

$$
\Delta_{L}^{T} \Phi=\lim _{n \rightarrow \infty} \frac{1}{n} \sum_{k=0}^{n-1} \widetilde{D}_{\zeta_{k}}^{2} \Phi
$$

if the limit exists in $(\mathcal{S})^{*}$. We denote the set of all functionals $\Phi$ such that $\Delta_{L}^{T} \Phi$ exists in $(\mathcal{S})^{*}$ and $S[\Phi](\eta)=0$ for any $\eta \in \mathcal{S}_{\mathbf{C}}$ with supp $\eta \subset T^{c}$ by $\mathcal{D}_{L}^{T}$. From now on, we fix an interval $T$ and use the notation $\Delta_{L}$ instead of $\Delta_{L}^{T}$.

For $p \geq 1$ and $\Phi \in \mathcal{D}_{L}^{T}$, we define a $(-p)$-norm $\left|\|\cdot \mid\|_{-p}\right.$ by

$$
\||| \Phi\|_{-p}=\left(\sum_{k=0}^{\infty}\left\|\Delta_{L}^{k} \Phi\right\|_{-p}^{2}\right)^{1 / 2} \in[0, \infty]
$$

and let $\mathcal{K}_{T,-p}$ denote the set of Hida distributions $\Phi$ in $(\mathcal{S})_{-p}$ such that $\left.\||\Phi|\|\right|_{-p}<$ $\infty$. Denote the completion of $\mathcal{K}_{T,-p}$ with respect to the norm $\|\mid \cdot\| \|_{-p}$ by $\mathbf{D}_{-p}$. Then $\mathbf{D}_{-p}$ is a Hilbert space with the norm $\left|\left\|\cdot|\||_{-p}\right.\right.$. Since $\mathcal{K}_{T,-p}$ is dense in $\mathbf{D}_{-p}$ and $\left\|\left.\left|\Delta_{L} \Phi\right|\right|_{-p} \leq\right\| \| \Phi||_{-p}$ for all $\Phi \in \mathcal{K}_{T,-p}$, the Lévy Laplacian can be extended to an operator $\bar{\Delta}_{L}$ in $\mathcal{L}\left(\mathbf{D}_{-p}\right)$ such that $\left.\left\|\left|\bar{\Delta}_{L} \Phi\right|\right\|\right|_{-p} \leq\left\||| \Phi|\||_{-p}\right.$ for all $\Phi \in \mathbf{D}_{-p}$. We denote $\bar{\Delta}_{L}$ by the same notation $\Delta_{L}$ from now on. We put $\mathbf{D}=\bigcup_{p=1}^{\infty} \mathbf{D}_{-p}$ with the inductive limit topology. Then $\Delta_{L}$ is a continuous linear operator in $\mathcal{L}(\mathbf{D})$.

For $p \geq 1$, let $\mathbf{E}_{-p}$ denote the normed linear space consisting of Hida distributions $\Phi=\sum_{n=0}^{\infty} \Phi_{n}$ in $\mathbf{D}_{-p}$ such that $\Phi_{n} \in \mathcal{N}_{T} \cap H_{n}^{(-p)}$ (see description 1 below for the definition of $\left.\mathcal{N}_{T}\right)$ for every $n \in \mathbf{N} \cup\{0\}$ and $\sum_{n=0}^{\infty}||\left|\Phi_{n}\right|||_{-p}<\infty$ with the norm \|\|$\cdot\left\|\left.\right|_{*,-p}=\sum_{n=0}^{\infty}\right\|||(\cdot)_{n} \mid \|_{-p}$. Then $\Delta_{L}$ is a bounded linear operator in $\mathcal{L}\left(\mathbf{E}_{-p}\right)$ such that ||$\left|\Delta_{L} \Phi\right|\left\|\left.\right|_{*,-p} \leq|||\Phi|\right\|_{*,-p}$ for $\Phi \in \mathbf{E}_{-p}$. We put $\mathbf{E}=\bigcup_{p=1}^{\infty} \mathbf{E}_{-p}$. Then $\Delta_{L}$ is also a continuous linear operator in $\mathcal{L}(\mathbf{E})$.

For any $p \geq 1$, the space $\mathbf{E}_{-p}$ includes the following functionals:

1) Normal functionals: A Hida distribution $\Phi$ is said to be normal if its $S$ transform $S[\Phi]$ is given by a finite linear combination of

$$
\int_{T^{k}} f\left(u_{1}, \ldots, u_{k}\right) \xi\left(u_{1}\right)^{p_{1}} \cdots \xi\left(u_{k}\right)^{p_{k}} d u_{1} \cdots d u_{k}
$$

where $f \in L^{1}\left(T^{k}\right)$ and $p_{1}, \ldots, p_{k} \in \mathbf{N} \cup\{0\}, k \in \mathbf{N}$. These functionals play an important role in the study of polynomials in the infinite dimensional analysis. Let $\mathcal{N}_{T}$ denote the set of all normal functionals in $\mathcal{D}_{L}^{T}$.

2) Exponential functionals: A Hida distribution

$$
\Phi_{c} \equiv \mathcal{N} \exp \left[\frac{c}{2} \int_{T} x(u)^{2} d u\right], \mathcal{N}: \text { normalizing factor, for any } c<1 / 2,
$$

is an important example as an eigen-functional of $\Delta_{L}$. The $S$-transform of $\Phi_{c}$ is given by

$$
S\left[\Phi_{c}\right](\xi)=\exp \left[\frac{c}{2(1-c)} \int_{T} \xi(u)^{2} d u\right], \xi \in \mathcal{S}_{\mathbf{C}}
$$


We introduce an operator $I_{M} \in \mathcal{L}\left((\mathcal{S})^{*}\right)$ by

$$
I_{M} \Phi=\sum_{n=0}^{M} \Phi_{n} \text { for } \Phi=\sum_{n=0}^{\infty} \Phi_{n} .
$$

As in [3], the following result holds.

Theorem 1. For any $\Phi \in \mathbf{E}$ we have

$$
\Delta_{L} \Phi=\lim _{M \rightarrow \infty} \lim _{N \rightarrow \infty} \frac{1}{N|T|} \Delta_{G}\left(P_{N}\right) I_{M} \Phi \text { in }(\mathcal{S})^{*}
$$

However, the limit in Theorem 1 converges in a stronger sense. In fact, we have the following.

Theorem 2. For any $\Phi \in \mathbf{E}$ we have

$$
\Delta_{L} \Phi=\lim _{M \rightarrow \infty} \lim _{N \rightarrow \infty} \frac{1}{N|T|} \Delta_{G}\left(P_{N}\right) I_{M} \Phi \text { in } \mathbf{E} .
$$

Proof. Let $\Phi \in \mathbf{E}$ be given with $\Phi=\sum_{n=0}^{\infty} \Phi_{n}$. Then there exists $p \geq 1$ such that for each $n \in \mathbf{N} \cup\{0\}, \Phi_{n} \in \mathcal{N}_{T} \cap H_{n}^{(-p)}$. Now, we shall show that for each $n \in \mathbf{N} \cup\{0\}$

Note that

$$
\Delta_{L} \Phi_{n}=\lim _{N \rightarrow \infty}\left(\frac{1}{N|T|} \Delta_{G}\left(P_{N}\right)\right) \Phi_{n} \text { in } \mathbf{E}
$$

$$
\begin{aligned}
& \left.\left\|\Delta_{L} \Phi_{n}-\frac{1}{N|T|} \Delta_{G}\left(P_{N}\right) \Phi_{n}\right\|\right|_{*,-p} ^{2} \\
& =\left\|\Delta_{L} \Phi_{n}-\frac{1}{N|T|} \Delta_{G}\left(P_{N}\right) \Phi_{n}\right\| \|_{-p}^{2} \\
& =\sum_{k=0}^{[(n-2) / 2]}\left\|\Delta_{L}^{k}\left(\Delta_{L} \Phi_{n}-\frac{1}{N|T|} \Delta_{G}\left(P_{N}\right) \Phi_{n}\right)\right\|_{-p}^{2} .
\end{aligned}
$$

Since $\Delta_{G}\left(P_{N}\right) \Phi_{n} \in \mathcal{N}_{T}$, we can check that

$$
\Delta_{L}^{k} \Delta_{G}\left(P_{N}\right) \Phi_{n}=\Delta_{G}\left(P_{N}\right) \Delta_{L}^{k} \Phi_{n}
$$

for each $0 \leq k \leq[(n-2) / 2]$ and $n \in \mathbf{N} \cup\{0\}$ by the direct calculation. Hence we have

$$
\begin{aligned}
& \left\|\left|\Delta_{L} \Phi_{n}-\frac{1}{N|T|} \Delta_{G}\left(P_{N}\right) \Phi_{n} \|\right|_{*,-p}^{2}\right. \\
& =\sum_{k=0}^{[(n-2) / 2]}\left\|\Delta_{L}\left(\Delta_{L}^{k} \Phi_{n}\right)-\frac{1}{N|T|} \Delta_{G}\left(P_{N}\right)\left(\Delta_{L}^{k} \Phi_{n}\right)\right\|_{-p}^{2} .
\end{aligned}
$$

On the other hand, by Theorem 1 , for any $\Psi \in \mathcal{N}_{T}$ there exists $p \geq 1$ such that

$$
\lim _{N \rightarrow \infty}\left\|\Delta_{L} \Psi-\frac{1}{N|T|} \Delta_{G}\left(P_{N}\right) \Psi\right\|_{-p}=0 .
$$

Since $\Delta_{L}^{k} \Phi_{n} \in \mathcal{N}_{T}$, for any $0 \leq k \leq[(n-2) / 2]$ there exists $p_{k} \geq 1$ such that

$$
\lim _{N \rightarrow \infty}\left\|\Delta_{L}\left(\Delta_{L}^{k} \Phi_{n}\right)-\frac{1}{N|T|} \Delta_{G}\left(P_{N}\right)\left(\Delta_{L}^{k} \Phi_{n}\right)\right\|_{-p_{k}}=0
$$


Take $p=\max \left\{p_{k}: 0 \leq k \leq[(n-2) / 2]\right\}$. Then we have

$$
\lim _{N \rightarrow \infty}\left\|\left|\Delta_{L} \Phi_{n}-\frac{1}{N|T|} \Delta_{G}\left(P_{N}\right) \Phi_{n} \|\right|_{*,-p}=0 .\right.
$$

Since $I_{M} \Phi=\sum_{n=0}^{M} \Phi_{n}$ for any $M \in \mathbf{N} \cup\{0\}$, we obtain that

$$
\begin{aligned}
\Delta_{L}\left(I_{M} \Phi\right) & =\sum_{n=0}^{M} \lim _{N \rightarrow \infty}\left(\frac{1}{N|T|} \Delta_{G}\left(P_{N}\right)\right)\left(\Phi_{n}\right) \\
& =\lim _{N \rightarrow \infty}\left(\frac{1}{N|T|} \Delta_{G}\left(P_{N}\right)\right)\left(I_{M} \Phi\right) \text { in } \mathbf{E} .
\end{aligned}
$$

Hence, by $\Delta_{L} \in \mathcal{L}(\mathbf{E})$, we have

$$
\lim _{M \rightarrow \infty} \lim _{N \rightarrow \infty}\left(\frac{1}{N|T|} \Delta_{G}\left(P_{N}\right)\right)\left(I_{M} \Phi\right)=\lim _{M \rightarrow \infty} \Delta_{L}\left(I_{M} \Phi\right)=\Delta_{L} \Phi \text { in } \mathbf{E} .
$$

Thus the proof is completed.

By induction, Theorem 2 implies the following:

Corollary 3. For any $\Phi \in \mathbf{E}$ and $\ell \in \mathbf{N} \cup\{0\}$, we have

$$
\Delta_{L}^{\ell} \Phi=\lim _{M \rightarrow \infty} \lim _{N \rightarrow \infty}\left(\frac{1}{N|T|}\right)^{\ell}\left(\Delta_{G}\left(P_{N}\right)\right)^{\ell}\left(I_{M} \Phi\right) \text { in } \mathbf{E} .
$$

4. A Relation BETWEen GROUPS GENERATED BY INFINITE DIMENSIONAL LAPLACIANS

For $K \in \mathcal{L}\left(\mathcal{S}_{\mathbf{C}}^{*}, \mathcal{S}_{\mathbf{C}}\right)$, we define a group $e^{z \Delta_{G}(K)}, z \in \mathbf{C}$, by

$$
e^{z \Delta_{G}(K)}=\lim _{n \rightarrow \infty} \sum_{k=0}^{n} \frac{z^{k}}{k !}\left(\Delta_{G}(K)\right)^{k} \in \mathcal{L}\left((\mathcal{S})^{*}\right) .
$$

We also define a $C_{0}$-group $e^{z \Delta_{L}}, z \in \mathbf{C}$, by

$$
e^{z \Delta_{L}}=\lim _{n \rightarrow \infty} \sum_{k=0}^{n} \frac{z^{k}}{k !} \Delta_{L}^{k} \in \mathcal{L}(\mathbf{D})
$$

It is easily checked that $e^{z \Delta_{L}}$ is in $\mathcal{L}(\mathbf{E})$ and for any $\Phi \in \mathbf{E}$ and $z \in \mathbf{C}$ there exists $p \geq 1$ such that ||$\left|e^{z \Delta_{L}} \Phi\right| \|\left.\right|_{*,-p} \leq e^{|z|}|||\Phi|||_{*,-p}$. Then we get the following.

Theorem 4. For any $\Phi \in \mathbf{E}$ and $z \in \mathbf{C}$, we have

$$
e^{z \Delta_{L}} \Phi=\lim _{M \rightarrow \infty} \lim _{N \rightarrow \infty} e^{z /(N|T|) \Delta_{G}\left(P_{N}\right)} I_{M} \Phi \text { in } \mathbf{E}
$$


Proof. Let $\Phi=\sum_{n=0}^{\infty} \Phi_{n} \in \mathbf{E}$. Then there exists some $p \geq 1$ such that $\Phi$ is in $\mathbf{E}_{-p}$. We have the following estimation:

$$
\begin{aligned}
& \|\left.\left|e^{z /(N|T|) \Delta_{G}\left(P_{N}\right)} I_{M} \Phi-e^{z \Delta_{L}} \Phi\right|\right|_{-p} \\
& \leq \sum_{\ell=0}^{\infty} \frac{|z|^{\ell}}{\ell !}\left|\left\|\left(\frac{1}{N|T|}\right)^{\ell}\left(\Delta_{G}\left(P_{N}\right)\right)^{\ell} I_{M} \Phi-\Delta_{L}^{\ell} \Phi|\||_{-p}\right.\right. \\
& \leq \sum_{\ell=0}^{\infty} \frac{|z|^{\ell}}{\ell !}\left[\sum_{k=0}^{M}\|\|\left(\frac{1}{N|T|}\right)^{\ell}\left(\Delta_{G}\left(P_{N}\right)\right)^{\ell} \Phi_{k}-\Delta_{L}^{\ell} \Phi_{k} \|\left.\right|_{-p}\right. \\
& \left.+\left.\left\|\Delta_{L}^{\ell} \sum_{k=M+1}^{\infty} \Phi_{k}\right\|\right|_{-p}\right] \\
& =\sum_{\ell=0}^{[M / 2]} \frac{|z|^{\ell}}{\ell !} \sum_{k=0}^{M}\|\|\left(\frac{1}{N|T|}\right)^{\ell}\left(\Delta_{G}\left(P_{N}\right)\right)^{\ell} \Phi_{k}-\Delta_{L}^{\ell} \Phi_{k} \|\left.\right|_{-p} \\
& +\sum_{\ell=0}^{\infty} \frac{|z|^{\ell}}{\ell !}\left\|\left|\Delta_{L}^{\ell} \sum_{k=M+1}^{\infty} \Phi_{k} \|\right|_{-p} .\right.
\end{aligned}
$$

By Corollary 3 , for any $\ell$ and $k \in \mathbf{N} \cup\{0\}$

$$
\lim _{N \rightarrow \infty}\left\|\left(\frac{1}{N|T|}\right)^{\ell}\left(\Delta_{G}\left(P_{N}\right)\right)^{\ell} \Phi_{k}-\Delta_{L}^{\ell} \Phi_{k}\right\| \|_{-p}=0 .
$$

Hence we have

$$
\lim _{M \rightarrow \infty} \lim _{N \rightarrow \infty} \sum_{\ell=0}^{[M / 2]} \frac{|z|^{\ell}}{\ell !} \sum_{k=0}^{M}\left|\left\|\left(\frac{1}{N|T|}\right)^{\ell}\left(\Delta_{G}\left(P_{N}\right)\right)^{\ell} \Phi_{k}-\Delta_{L}^{\ell} \Phi_{k}\right\|\right|_{-p}=0 .
$$

Since

$$
\left.\left\|\Delta_{L}^{\ell} \sum_{k=M+1}^{\infty} \Phi_{k}\right\|\left\|_{-p} \leq \sum_{k=M+1}^{\infty}\right\|\left\|\Phi_{k}\right\|\right|_{-p} \leq \sum_{k=0}^{\infty}\left\||| \Phi_{k}\right\| \|_{-p}<\infty
$$

we have

$$
\lim _{M \rightarrow \infty} \sum_{\ell=0}^{\infty} \frac{|z|^{\ell}}{\ell !}\left\|\left|\Delta_{L}^{\ell} \sum_{k=M+1}^{\infty} \Phi_{k} \|\right|_{-p}=0\right.
$$

Therefore we obtain

$$
\lim _{M \rightarrow \infty} \lim _{N \rightarrow \infty}\left\||| e^{(z /(N|T|)) \Delta_{G}\left(P_{N}\right)} I_{M} \Phi-e^{z \Delta_{L}} \Phi \mid\right\|_{-p}=0 .
$$

This implies the assertion.

\section{Fundamental solution associated with the LÉvy Laplacian}

The Kubo-Yokoi delta function depending on $T$ is a generalized white noise functional $\widetilde{\delta}_{x}, x \in \mathcal{S}^{*}$ whose $S$-transform is given by

$$
S\left[\widetilde{\delta}_{x}\right](\xi)=\exp \left[\int_{T} x(u) \xi(u) d u-\frac{1}{2} \int_{T} \xi(u)^{2} d u\right], \xi \in E
$$


The function $\widetilde{\delta} \equiv \widetilde{\delta}_{0}$ is also given by the limit of $\Phi_{c}$ (in $\S 3$, description 2 ) in $(\mathcal{S})^{*}$ as $c \rightarrow \infty$.

Put $L=\partial / \partial t-\alpha \Delta_{L}$. Consider the fundamental solution of $L$, i.e. a solution of

$$
L \Phi(t, x)=\delta(t) \otimes \widetilde{\delta}(x) .
$$

Lemma 5. For any $t \geq 0$, there exists $\Phi(t, x) \in \mathcal{S}_{\mathbf{C}}^{*} \otimes(\mathcal{S})^{*}$ such that

$$
\left\langle\Phi(t, \cdot), \eta \otimes \phi_{\xi}\right\rangle=e^{-\alpha t} \int_{0}^{t} \eta(u) e^{\alpha u} d u S[\widetilde{\delta}](\xi), \eta, \xi \in \mathcal{S}_{\mathbf{C}} .
$$

Proof. Let $F_{t}(u)=1_{[0, t]}(u) e^{-\alpha(t-u)}$ for $t \geq 0$ and $u \in \mathbf{R}$, and set $\Phi(t, x)=F_{t} \otimes \widetilde{\delta}(x)$. Then $\Phi(t, x) \in \mathcal{S}_{\mathbf{C}}^{*} \otimes(\mathcal{S})^{*}$ and also

$$
\left\langle\Phi(t, x), \eta \otimes \phi_{\xi}\right\rangle=\left\langle F_{t}, \eta\right\rangle\left\langle\widetilde{\delta}, \phi_{\xi}\right\rangle .
$$

This implies (5.2).

Theorem 6. For any $t \geq 0, \Phi(t, x)$ given as in Lemma 5 is a solution of (5.1).

Proof. By direct calculation, we have

$$
\begin{aligned}
\left\langle\left(\frac{\partial}{\partial t}-\alpha \Delta_{L}\right) \Phi(t, x), \eta \otimes \phi_{\xi}\right\rangle & =\left(\frac{\partial}{\partial t}-\alpha \widetilde{\Delta}_{L}\right) e^{-\alpha t} \int_{0}^{t} \eta(u) e^{\alpha u} d u S[\widetilde{\delta}](\xi) \\
& =\eta(t) S[\widetilde{\delta}](\xi) \\
& =\left\langle\delta(t) \otimes \widetilde{\delta}, \eta \otimes \phi_{\xi}\right\rangle
\end{aligned}
$$

where $\widetilde{\Delta}_{L}$ is defined by

$$
\widetilde{\Delta}_{L} S[\Phi](\xi)=\lim _{N \rightarrow \infty} \frac{1}{N} \sum_{k=0}^{N-1} S[\Phi]^{\prime \prime}(\xi)\left(\zeta_{k}, \zeta_{k}\right), \quad \Phi \in(\mathcal{S})^{*}, \xi \in \mathcal{S}_{\mathbf{C}}
$$

if the limit exists in $S\left[(\mathcal{S})^{*}\right]$ (see [9, 16]).

\section{CONCLUDing REMARKS}

Let $\overline{\mathbf{E}}_{-p}$ be the completion of $\mathbf{E}_{-p}$ with respect to the norm $\||\cdot|\| \|_{*,-p}$ for every $p \geq 1$. Then this becomes a Banach space with norm $\mid\|\cdot\| \|_{*,-p}$. Since for any $p \geq 1$ the Laplacian $\Delta_{L}$ is in $\mathcal{L}\left(\mathbf{E}_{-p}\right)$, it can be extended to a continuous linear operator in $\mathcal{L}\left(\overline{\mathbf{E}}_{-p}\right)$. Our next problem is to discuss the operator in $\mathcal{L}\left(\overline{\mathbf{E}}_{-p}\right)$ with the stochastic process associated with the operator. The results of this problem will appear elsewhere.

\section{ACKNOWLEDGEMENTS}

This work was supported, in part, by the Research Project "Quantum Information Theoretical Approach to Life Science" for the Academic Frontier in Science, promoted by the Ministry of Education in Japan and was also supported by Korea Research Foundation (KRF-2000-015-DP0016). U.C. Ji was partially supported by KOSEF. K. Saitô was partially supported by JMESSC Grant-in-Aid for Scientific Research (C)(2) 11640139. The authors are grateful for their support. 


\section{REFERENCES}

1. Accardi, L.: Yang-Mills equations and the Lévy Laplacian, in "Dirichlet Forms and Stochastic Processes", (Z.M. Ma, M. Röckner and J.A. Yan eds.) de Gruyter, 1-24, 1995. MR 97c:46093

2. Chung, D. M. and Ji, U. C.: Transforms on white noise functionals with their applications to Cauchy problems, Nagoya Math J. 147 (1997), 1-23. MR 99b:60098

3. Chung, D. M., Ji, U. C. and Saitô, K.: Cauchy problems associated with the Lévy Laplacian in white noise analysis, Infinite Dimensional Analysis, Quantum Probability and Related Topics 2, No.1 (1999), 131-153.

4. Gross, L.: Abstract Wiener spaces; Proc. 5th Berkeley Symp. Math. Stat. Probab. 2 (1965), 31-42. Berkeley: Univ. Berkeley. MR 35:3027

5. Gross, L.: Potential theory on Hilbert space; J. Func. Anal. 1 (1967), 123-181. MR 37:3331

6. Hida, T.: "Analysis of Brownian Functionals", Carleton Math. Lecture Notes, No.13, Carleton University, Ottawa, 1975. MR 56:9715

7. Hida, T.: A role of the Lévy Laplacian in the causal calculus of generalized white noise functionals, in "Stochastic Processes A Festschrift in Honour of G. Kallianpur " (S. Cambanis et al. Eds.) Springer-Verlag, 1992. MR 97j:6049

8. Hida, T., Kuo, H. - H. and Obata, N.: Transformations for white noise functionals, J. Funct. Anal. 111 (1990), 259-277. MR 93m:46042

9. Hida, T., Kuo, H. - H., Potthoff, J. and Streit, L.: "White Noise: An Infinite Dimensional Calculus", Kluwer Academic, 1993. MR 95f:60046

10. Hida, T. and Saitô, K.: White noise analysis and the Lévy Laplacian, in "Stochastic Processes in Physics and Engineering" (S. Albeverio et al. Eds.), 177-184, 1988. MR 89m:60092

11. Hida, T., Obata, N. and Saitô, K.: Infinite dimensional rotations and Laplacian in terms of white noise calculus, Nagoya Math. J. 128 (1992), 65-93. MR 94a:60119

12. Itô, K.: Stochastic analysis in infinite dimensions, in "Proc. International conference on stochastic analysis", Academic Press, Evanston, 187-197, 1978. MR 80k:60073

13. Kubo, I.: A direct setting of white noise calculus, in: Stochastic analysis on infinite dimensional spaces, Pitman Research Notes in Mathematics Series, 310 (1994), 152-166. MR 98b:60072

14. Kubo, I. and Takenaka, S.: Calculus on Gaussian white noise I, II, III and IV, Proc. Japan Acad. 56A (1980), 376-380; 56A (1980), 411-416; 57A (1981), 433-436; 58A (1982), 186-189. MR 84d:60062 MR 84d:60063a MR 84d:60063b; MR 84m:60100

15. Kuo, H. - H.: On Laplacian operators of generalized Brownian functionals, in "Lecture Notes in Math." 1203, Springer-Verlag, 119-128, 1986. MR 88e:60078

16. Kuo, H. - H.: White noise distribution theory, CRC Press (1996). MR 97m:60056

17. Kuo, H. - H., Obata, N. and Saitô, K.: Lévy Laplacian of generalized functions on a nuclear space, J. Funct. Anal. 94 (1990), 74-92. MR 91m:46061

18. Lee, Y.-J.: Integral transforms of analytic functions on abstract Wiener spaces, J. Funct. Anal. 47 (1983), 153-164. MR 84j:28021

19. Lee, Y.-J.: Unitary operators on the space of $L^{2}$-functions over abstract Wiener spaces, Soochow J. Math. 13 (1987), 165-174. MR 89h:47045

20. Lévy, P.: "Lecons d'analyse fonctionnelle", Gauthier-Villars, Paris, 1922.

21. Obata, N.: A characterization of the Lévy Laplacian in terms of infinite dimensional rotation groups, Nagoya Math. J. 118 (1990), 111-132. MR 91m:46063

22. Obata, N.: "White Noise Calculus and Fock Space," Lecture Notes in Mathematics 1577, Springer-Verlag, 1994. MR 96e:60061

23. Potthoff, J. and Streit, L.: A characterization of Hida distributions, J. Funct. Anal. 101 (1991), 212-229. MR 93a:46078

24. Saitô, K.: Itô's formula and Lévy's Laplacian I and II, Nagoya Math. J. 108 (1987), 67-76; 123 (1991), 153-169. MR 89f:60042 MR 93c:60057

25. Saitô, K.: A group generated by the Lévy Laplacian and the Fourier-Mehler transform, in: Stochastic analysis on infinite dimensional spaces, Pitman Research Notes in Mathematics Series 310 (1994), 274-288. MR 97i:60107

26. Saitô, K.: A $\left(C_{0}\right)$-group generated by the Lévy Laplacian, Journal of Stochastic Analysis and Applications 16, No. 3 (1998), 567-584. MR 99g:60109 
27. Saitô, K.: A $\left(C_{0}\right)$-group generated by the Lévy Laplacian II, Infinite Dimensional Analysis, Quantum Probability and Related Topics 1, No. 3 (1998), 425-437. MR 99k:46074

28. Yosida, K.: "Functional Analysis 3rd Edition", Springer-Verlag, 1971. MR 39:741 (review of 2nd edition)

Department of Mathematics, Sogang University, Seoul 121-742, Korea

E-mail address: dmchung@ccs.sogang.ac.kr

Department of Mathematics, Sogang University, Seoul 121-742, Korea

E-mail address: ucji@nuri.net

Department of Information Sciences, Meijo University, Tempaku, Nagoya 468-8502, JAPAN

E-mail address: ksaito@meijo-u.ac.jp 